\title{
Variation in Practice Patterns of Korean Radiation Oncologists for Spine Metastasis between 2009 and 2014
}

\author{
Jeong II Yu, MD' \\ Hee Chul Park, MD, PhD',2 \\ Yong Chan Ahn, MD, PhD ${ }^{1,2}$ \\ Yoonsun Chung, $\mathrm{PhD}^{1}$ \\ Woong Sub Koom, MD, PhD 3 \\ Si Yeol Song, MD, PhD ${ }^{4}$
}

\begin{abstract}
Purpose
The Korean Society of Radiation Oncologists (KOSRO) conducted the Patterns of Care Study (PCS) of radiotherapy (RT) for spine metastases in 2009. The current study was conducted to investigate current practice patterns and compare them with the results of the PCS.
\end{abstract}

\section{Materials and Methods}

The survey questionnaire was composed of 10 questions regarding general information and seven questions for each of two clinical scenarios.

\section{Results}

Fifty-four members of the KOSRO answered at least one question on the web-based questionnaire. The yearly number of patients treated who underwent palliative spine RT was greater than 200 in 14 (25.9\%), 51 to 100 in 13 (24.1\%), and 31 to 50 in 11 respondents (20.4\%). Scenario 1 described a patient presenting with cord compressive spine metastasis in multiple bones and liver metastasis from non-small cell lung cancer. Thirty gray (Gy) in 10 fractions was chosen by 35 respondents (64.8\%). Scenario 2 described a case of a single spine metastasis without progression after targeted therapy. Thirty Gy in 10 fractions was chosen by 19 respondents (35.2\%), and a single fraction or less than four fractions of stereotactic ablative radiotherapy (SABR) were selected by 18 respondents (33.3\%). When compared with the 2009 PCS, practice patterns of Korean radiation oncologists had not changed significantly over 5 years, except that SABR emerged as a new treatment modality in the selected population.

\section{Conclusion}

The 2014 PCS demonstrated that multiple fraction RT is still preferred in a considerable proportion of Korean radiation oncologists.
Correspondence: Hee Chul Park, MD, PhD Department of Radiation Oncology, Samsung Medical Center,

Sungkyunkwan University School of Medicine, 81 Irwon-ro, Gangnam-gu, Seoul 06351, Korea Tel: 82-2-3410-2605

Fax: 82-2-3410-2619

E-mail: hee.ro.park@samsung.com

Received June 11, 2015

Accepted November 7, 2015

Published Online December 2, 2015

\section{Key words}

Data collection, Spine, Neoplasm metastasis, Radiotherapy, Physician's practice patterns, Korea

\section{Introduction}

Spine metastasis is highly problematic in clinical oncology practice [1]. A tremendous survival difference can be seen in patients with spine metastasis according to disease extent, histologic type, treatment method, performance status, and duration of disease free survival [2-6]. Because of the diverse outcomes of spine metastases based on different disease presentations, there is considerable heterogeneity in practice patterns for spine metastasis, even when focusing only on patterns of radiation oncology practice [7-10]. Furthermore, survival duration of patients with distant metastasis has been continuously extended due to recent developments in sys- 
temic, loco-regional, and supportive care [11,12]. For these reasons, single-fraction radiotherapy (RT) is still underused globally, although several randomized studies have indicated that single-fraction RT is as effective as multiple fractions [13-15].

A previous study of practice patterns of Korean radiation oncologists for spine metastasis was conducted in October 2009 with results published in December 2013 [16]. In that study, most Korean radiation oncologists preferred multiple fractionations over single-fraction RT, findings similar to those of patterns of care studies (PCS) conducted in many other countries.

The current study was planned to investigate the current practice patterns of RT for spine metastasis and to compare these patterns with the results of the 2009 Korean Society of Radiation Oncologists (KOSRO) PCS on spine metastasis.

\section{Materials and Methods}

\section{Participants and survey}

The subjects of the current PCS were practicing KOSRO members, who completed an anonymous web-based survey using Google Docs (Mountain View, CA) from September 2 to September 25, 2014. We requested a response from one representative radiation oncologist from each institution. Of 81 Korean institutions practicing RT (list of the institutions is included in the Supplementary Table 1) who received the questionnaire at the time of the survey, 54 radiation oncologists responded, with a nationwide response rate of $66.7 \%$.

\section{Questionnaire}

A 24-item questionnaire was created through discussion with four KOSRO radiation oncologists (Supplementary Fig. S1). The questionnaire included questions addressing: (1) general characteristics of the respondents including factors that could influence practice patterns; and (2) two clinical scenarios, which were developed based on typical cases with a significantly different prognosis. A further scenario regarding local tumor progression at the same site after RT was added for evaluation of patterns of re-irradiation.

\section{Statistical analysis}

Data analysis was performed using the IBM SPSS Statistic ver. 22.0 software for Windows (IBM Co., Armonk, NY). The chi-square test or Fisher exact test was used for comparisons between groups, and the Mann-Whitney test was used in the comparison of patterns of fractionation and fraction size between 2009 and 2014. $p<0.05$ was considered statistically significant.

\section{Results}

\section{Respondents}

Among the 54 respondents representing their institutions, 47 respondents $(87.0 \%)$ worked at educational/university hospitals. Five $(9.3 \%)$ worked at public hospitals and the other two $(3.7 \%)$ were employed in private hospitals.

The number of patients receiving daily RT was 51 to 100 in 18 respondents $(33.3 \%), 50$ or less in 15 respondents $(27.8 \%)$, and more than 300 in nine respondents $(16.7 \%)$. The yearly number of patients receiving palliative spine RT was more than 200 in 14 respondents (25.9\%), 51 to 100 in 13 respondents $(24.1 \%)$, and 31 to 50 in 11 respondents (20.4\%). The period of expert radiation oncology practice was 5 to 9 years in 21 respondents (38.9\%), 10 to 14 in 13 respondents $(24.1 \%)$, and 15 or more in 20 respondents (37.0\%).

The specific characteristics of the respondents are summarized in Table 1.

\section{Scenario 1}

This case describes typical spine metastasis, and the expected 1-year survival is $25 \%$ based on the results of previous studies.

In the first question of scenario 1, a total of 11 different RT schedules were suggested (range, $8 \mathrm{~Gy} / 1$ fraction to 45 Gy / 25 fractions with gross tumor boost). Nine respondents $(16.7 \%)$ answered that they preferred to use five or fewer fractions, and the other $41(75.9 \%)$ preferred 10 or more fractions. Four respondents did not answer this question. In the second question, the preferred RT technique was a single postero-anterior (PA) field in 14 respondents $(25.9 \%)$, parallel opposite antero-posterior (AP)/PA fields in 15 respondents $(27.8 \%)$, three-dimensional conformal RT (3D-CRT) in $13(24.1 \%)$, and intensity-modulated RT (IMRT) in two respondents (3.7\%). The other 10 respondents did not answer this question. In the third question, except for three nonresponders, 37 of 51 respondents $(68.5 \%)$ replied that steroid treatment was required in this case.

The other three questions were about the presumed re-irradiation situation in the same case. Among 50 respondents who answered this question, 31 (57.4\%) recommended re-irradiation in the fourth question. In the fifth question, with more diverse RT schedules, 17 respondents suggested 
Table 1. Characteristics of respondents

\begin{tabular}{|lc|}
\hline Variable & No. of responders (\%) \\
\hline Practice type & \\
\hline Education/University & $47(87.0)$ \\
\hline Public & $5(9.3)$ \\
\hline Private & $2(3.7)$ \\
\hline Average No. of daily patients & \\
\hline$\leq 50$ & $15(27.8)$ \\
\hline $51-100$ & $18(33.3)$ \\
\hline $101-200$ & $5(9.3)$ \\
\hline $201-300$ & $5(9.3)$ \\
\hline$>300$ & $9(16.7)$ \\
\hline Not answered & $2(3.7)$ \\
\hline $\begin{array}{c}\text { Average No. of annual patients received } \\
\text { palliative spine RT }\end{array}$ \\
\hline$<30$ & \\
\hline $31-50$ & $7(13.0)$ \\
\hline $51-100$ & $11(20.4)$ \\
\hline $101-200$ & $13(24.1)$ \\
\hline$>200$ & $7(13.0)$ \\
\hline Not answered & $14(25.9)$ \\
\hline No. of co-workers as radiation & $2(3.7)$ \\
\hline oncologist specialists & \\
\hline 1 & \\
\hline 2 & $7(13.0)$ \\
\hline $3-5$ & $12(22.2)$ \\
\hline $6-10$ & $20(37.0)$ \\
\hline$>10$ & $10(18.5)$ \\
\hline Period of practicing radiation & $5(9.3)$ \\
\hline oncologist specialist (yr) & \\
\hline $5-9$ & $21(38.9)$ \\
\hline $10-14$ & $13(24.1)$ \\
\hline Specialty of radiation oncology & $20(37.0)$ \\
\hline (repeated choice) & \\
\hline Head and neck & $24(46.7)$ \\
\hline Central nervous system & $15(27.0 .3)$ \\
\hline Lung and thorax & $33(61.1)$ \\
\hline Breast & $21(38.9)$ \\
\hline Gastrointestinal & $26(48.1)$ \\
\hline Genitourinary & $21(38.9)$ \\
\hline Gynecology & $22(40.7)$ \\
\hline Pediatric & \\
\hline Hematologic & \\
\hline Benign or others & \\
\hline & \\
\hline
\end{tabular}

re-irradiation (range, $8 \mathrm{~Gy} / 1$ fraction to $30 \mathrm{~Gy} / 15$ fractions), and 16 respondents did not answer this question. There was a preference for smaller fractions in this re-irradiation situation; $20 \mathrm{~Gy} / 5$ fractions $(7 / 54,13.0 \%)$ and $8 \mathrm{~Gy} / 1$ fraction $(6 / 54,11.1 \%)$ were the most commonly selected schemes.
Regarding the technical aspect of the sixth question, 23 respondents $(42.6 \%)$ preferred a more complicated technique such as IMRT or stereotactic body ablative RT (SABR). Among the 42 respondents who answered the seventh question, $35(83.3 \%)$ stated that steroid treatment should be recommended in this case.

\section{Scenario 2}

This scenario describes a case of spine metastasis with good prognosis and an expected 1-year survival of more than $50 \%$.

For this favorable prognostic case, a total of 14 different RT schedules were suggested in the first question (range, $20 \mathrm{~Gy} / 4$ fractions to $60 \mathrm{~Gy} / 24$ fractions). Ten respondents $(18.5 \%)$ recommended using a single fraction. Twenty-three respondents preferred less than 10 fractions and the other 20 respondents chose 10 or more fractions; one respondent failed to complete this question. The preferred RT technique of the second question was a single PA field in five respondents $(9.3 \%)$, parallel opposite AP / PA fields in 12 respondents $(22.2 \%)$, and 3D-CRT in 13 respondents $(24.1 \%)$. IMRT or SABR was preferred by 20 respondents $(37.0 \%)$. In the third question, 10 respondents (18.5\%) suggested that steroid treatment was required in this case, except one who did not answer.

In the three questions regarding re-irradiation after local progression in the same case, among 53 respondents, 32 respondents $(57.4 \%)$ recommended re-irradiation. Fourteen RT schedules were suggested in the presumed re-irradiation situation. Single 8 Gy was the most preferred scheme $(7 / 54$, $13.0 \%$ ), and 11 respondents did not answer this question. In the technical aspect, 28 respondents $(51.9 \%)$ preferred IMRT or SABR as a re-irradiation technique. Twenty-nine respondents recommended steroid treatment.

\section{Related factors for the pattern of fractionation}

The general characteristics of the respondents, which could potentially be related to the chosen fractionation patterns, were analyzed and are shown in Table 2. In scenario 1, there were no significantly related factors. However, respondents who worked with five or more radiation oncologist specialists at their institutions were more than two times as likely to use fewer than five fractions $(\mathrm{p}=0.03)$. Physicians with a specialty in the lung or thorax showed less preference for a short fraction scheme $(\mathrm{p}=0.047)$ in scenario 2 .

\section{Related factors for the pattern of RT technique}

The general characteristics of the respondents, which could potentially influence the selection of RT technique, were 
Table 2. Factors potentially related to the pattern of fractionation

\begin{tabular}{|c|c|c|c|c|c|c|}
\hline \multirow{2}{*}{ Factor } & \multicolumn{3}{|c|}{ Scenario 1} & \multicolumn{3}{|c|}{ Scenario 2} \\
\hline & $\leq 5 \mathrm{fx}$ & $>5 \mathrm{fx}$ & p-value & $\leq 5 \mathrm{fx}$ & $>5 \mathrm{fx}$ & p-value \\
\hline \multicolumn{7}{|l|}{ Type of practice } \\
\hline Education/University & $9(20.0)$ & $36(80.0)$ & 0.65 & $19(41.3)$ & $27(58.7)$ & 0.82 \\
\hline Public & 0 & $5(100)$ & & $3(60.0)$ & $2(40.0)$ & \\
\hline Private & 0 & $1(100)$ & & $1(50.0)$ & $1(50.0)$ & \\
\hline \multicolumn{7}{|c|}{ On average number of daily RT patients } \\
\hline$>200$ & $2(15.4)$ & $11(84.6)$ & $>0.99$ & $8(61.5)$ & $5(38.5)$ & 0.11 \\
\hline$\leq 200$ & $7(19.4)$ & $29(80.6)$ & & $13(34.2)$ & $25(65.8)$ & \\
\hline \multicolumn{7}{|c|}{ On average number of annual palliative spine RT } \\
\hline$>100$ & $2(10.0)$ & $18(90.0)$ & 0.28 & $11(55.0)$ & $9(45.0)$ & 0.25 \\
\hline$\leq 100$ & $7(24.1)$ & $22(75.9)$ & & $11(35.5)$ & $20(64.5)$ & \\
\hline \multicolumn{7}{|c|}{ No. of dedicated radiation oncology specialist } \\
\hline$>5$ & $2(14.3)$ & $12(85.7)$ & $>0.99$ & $10(71.4)$ & $4(28.6)$ & 0.03 \\
\hline$\leq 5$ & $7(18.9)$ & $30(81.1)$ & & $13(33.3)$ & $26(66.7)$ & \\
\hline \multicolumn{7}{|c|}{ Period of practice as a radiation oncology specialist (yr) } \\
\hline$<15$ & $4(12.5)$ & $28(87.5)$ & 0.27 & $16(48.5)$ & $17(51.5)$ & 0.40 \\
\hline$\geq 15$ & $5(26.3)$ & $14(73.7)$ & & $7(35.0)$ & $13(65.0)$ & \\
\hline \multicolumn{7}{|c|}{ Type of specialty in radiation oncology } \\
\hline Lung/thorax & $5(16.1)$ & $26(83.9)$ & 0.72 & $10(31.3)$ & $22(68.8)$ & 0.047 \\
\hline Other & $4(20.0)$ & $16(80.0)$ & & $13(61.9)$ & $8(38.1)$ & \\
\hline \multicolumn{7}{|c|}{ Type of specialty in radiation oncology } \\
\hline Breast & $4(20.0)$ & $16(80.0)$ & 0.72 & $8(38.1)$ & $13(61.9)$ & 0.58 \\
\hline Other & $5(16.1)$ & $26(83.9)$ & & $15(46.9)$ & $17(53.1)$ & \\
\hline
\end{tabular}

Values are presented as number (\%). RT, radiotherapy.

analyzed and are shown in Table 3; there were no statistically significant factors in either scenario 1 or 2 . However, there was a tendency of using advanced technique in the respondents who worked with five or more radiation oncologist specialists.

\section{Changes in practice patterns between 2009 and 2014}

A direct comparison of changes between 2009 and 2014 is not possible because of the difference in clinical scenarios used for each survey; however, a comparison of practice patterns might be possible by categorizing scenarios by performance and life expectancy of the patient. Regarding the scenario of a patient with a short life expectancy (scenario 1), nine of 51 respondents $(17.6 \%)$ preferred five or fewer fractionations (Fig. 1A). In the previous study there were cases with a similar performance status to L3 spine metastasis with multiple liver and abdominal lymph nodes in small cell lung cancer; eight of $80(10.0 \%)$ answered that fewer than 10 fractions were adequate (Fig. 1B). And, the preferred number of fractions $(p=0.175)$ and fraction size $(p=0.197)$ were not significantly different between 2009 and 2014 in the case show- ing an unfavorable prognosis.

For the scenario of a patient with a favorable life expectancy (scenario 2), 23 of 53 respondents (43.4\%) preferred five or fewer fractions (Fig. 2A), while only six of 86 respondents $(7.0 \%)$ chose fewer than ten fractions in a similar case of breast cancer with T6 and 7 spine metastases only in the previous study (Fig. 2B). And, the preferred number of fractions and fraction size (both, $\mathrm{p}<0.001$ ) differed significantly between 2009 and 2014 in the case showing a favorable prognosis.

\section{Discussion}

In the current PCS that investigated current practice patterns and compared the results with those of a previous study on spine metastasis, we found that the practice patterns of Korean radiation oncologists for spine metastasis patients with a short life expectancy has not changed despite publication of the previous PCS. In the selected spine metas- 
Table 3. Factors potentially related to the pattern of RT technique

\begin{tabular}{|c|c|c|c|c|c|c|}
\hline \multirow{2}{*}{ Factor } & \multicolumn{3}{|c|}{ Scenario 1} & \multicolumn{3}{|c|}{ Scenario 2} \\
\hline & Low & High & p-value & Low & High & p-value \\
\hline \multicolumn{7}{|l|}{ Type of practice } \\
\hline Education/University & $38(97.4)$ & $1(2.6)$ & 0.052 & $23(54.8)$ & $19(45.2)$ & 0.65 \\
\hline Public & $4(100)$ & 0 & & $2(50.0)$ & $2(50.0)$ & \\
\hline Private & 0 & $1(100)$ & & $2(100)$ & 0 & \\
\hline \multicolumn{7}{|c|}{ On average number of daily RT patients } \\
\hline$>200$ & $12(100)$ & 0 & $>0.99$ & $5(45.5)$ & $6(54.5)$ & 0.48 \\
\hline$\leq 200$ & $28(93.3)$ & $2(6.7)$ & & $22(62.9)$ & $13(37.1)$ & \\
\hline \multicolumn{7}{|c|}{ On average number of annual palliative spine RT } \\
\hline$>100$ & $17(100)$ & 0 & 0.51 & $8(44.4)$ & $10(55.6)$ & 0.23 \\
\hline$\leq 100$ & $23(92.0)$ & $2(8.0)$ & & $18(64.3)$ & $10(35.7)$ & \\
\hline \multicolumn{7}{|c|}{ No. of dedicated radiation oncology specialists } \\
\hline$>5$ & $13(100)$ & 0 & $>0.99$ & $4(33.3)$ & $8(66.7)$ & 0.10 \\
\hline$\leq 5$ & $29(93.5)$ & $2(6.5)$ & & $23(63.9)$ & $13(36.1)$ & \\
\hline \multicolumn{7}{|c|}{ Period of practice as a radiation oncology specialist } \\
\hline$<15 \mathrm{yr}$ & $26(96.3)$ & $1(3.7)$ & $>0.99$ & $15(51.7)$ & $14(48.3)$ & 0.56 \\
\hline$\geq 15 \mathrm{yr}$ & $16(94.1)$ & $1(5.9)$ & & $12(63.2)$ & $7(36.8)$ & \\
\hline \multicolumn{7}{|c|}{ Type of specialty in radiation oncology } \\
\hline Lung/Thorax & $25(96.2)$ & $1(3.8)$ & $>0.99$ & $18(62.1)$ & $11(37.9)$ & 0.38 \\
\hline Other & $17(94.4)$ & $1(5.6)$ & & $9(47.4)$ & $10(52.6)$ & \\
\hline \multicolumn{7}{|c|}{ Type of specialty in radiation oncology } \\
\hline Breast & $15(93.8)$ & $1(6.3)$ & $>0.99$ & $15(71.4)$ & $6(28.6)$ & 0.08 \\
\hline Other & $27(96.4)$ & $1(3.6)$ & & $12(44.4)$ & $15(55.6)$ & \\
\hline
\end{tabular}

Values are presented as number (\%). RT, radiotherapy.

tasis case with expected long-term survival, however, there is a clear pattern change from greater fractionation to five or fewer fractions.

External beam RT has become an essential part of the management of painful, uncomplicated bone metastasis from almost all malignant diseases [17]. Many randomized studies have attempted to identify a more effective and convenient scheme of RT fractionation from ten to single [13-15]. These studies consistently showed that there was no difference in the pain reduction rate according to the number of fractions. Short course schedules were more cost-effective than a protracted course, even when considering the higher re-irradiation rate [18]. Considering the convenience, time-saving nature, and cost-effectiveness of smaller fractionation schemes, a single fraction might be a reasonable approach to management of bone metastasis.

As mentioned above, however, the re-irradiation rate was consistently higher in short-course $\mathrm{RT}$, and these results may reflect that a higher proportion of patients who received a short course of RT were suffering from pain and for a longer duration. If the expected survival time is extended, the proportion and duration would be increased. A reliable assump- tion of survival expectancy in spine metastasis, therefore, could be the first step in appropriate decision making. From this point of view, many randomized controlled trials conducted before 2000 have some limitations, because all spine metastases were enrolled without categorizing known prognostic factors such as performance status, primary tumor location, and disease extent [13-15]. In addition, the reported median survival duration was primarily less than 12 months with a median time to treatment failure of less than six months [14]. Because survival duration varies according to patient characteristics and disease status, from less than three months to more than 2 years [19-21], an adaptation of short fractionation without considering the patient's situation could be inappropriate.

Because of the many factors and conditions that should be considered in the management of spine metastasis, there is considerable variation in the pattern of clinical practice in Korean radiation oncologists as well as worldwide $[7,16]$. The diversity of practice patterns was re-affirmed in the current study; 30 Gy in 10 fractions is still the most preferred regimen, particularly in scenario 1 , which had a less than 1-year expected survival, in which more than $70 \%$ of resp- 

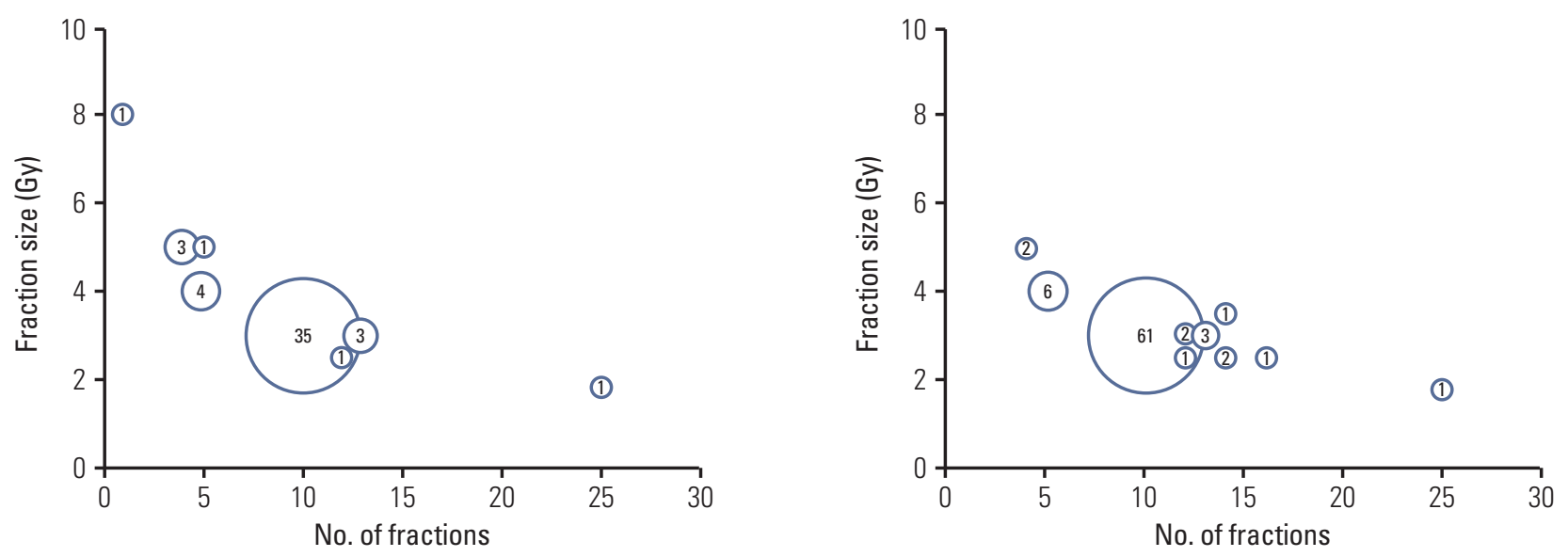

Fig. 1. Practice pattern changes from 2009 to 2014 in similar scenarios with a short life expectancy. Preference for radiotherapy fractionation in a case with a Karnofsky performance status of 70 and T6-10 level spine metastases with multiple liver metastases in the current study (A). A similar case in the 2009 study with a similar performance status with L3 spine metastases and multiple liver and abdominal lymph nodes metastases (B) is presented. Many Korean radiation oncologists responded that they preferred the multiple fractionation regimen; these results were similar between the 2009 and 2014 studies.

\section{A}
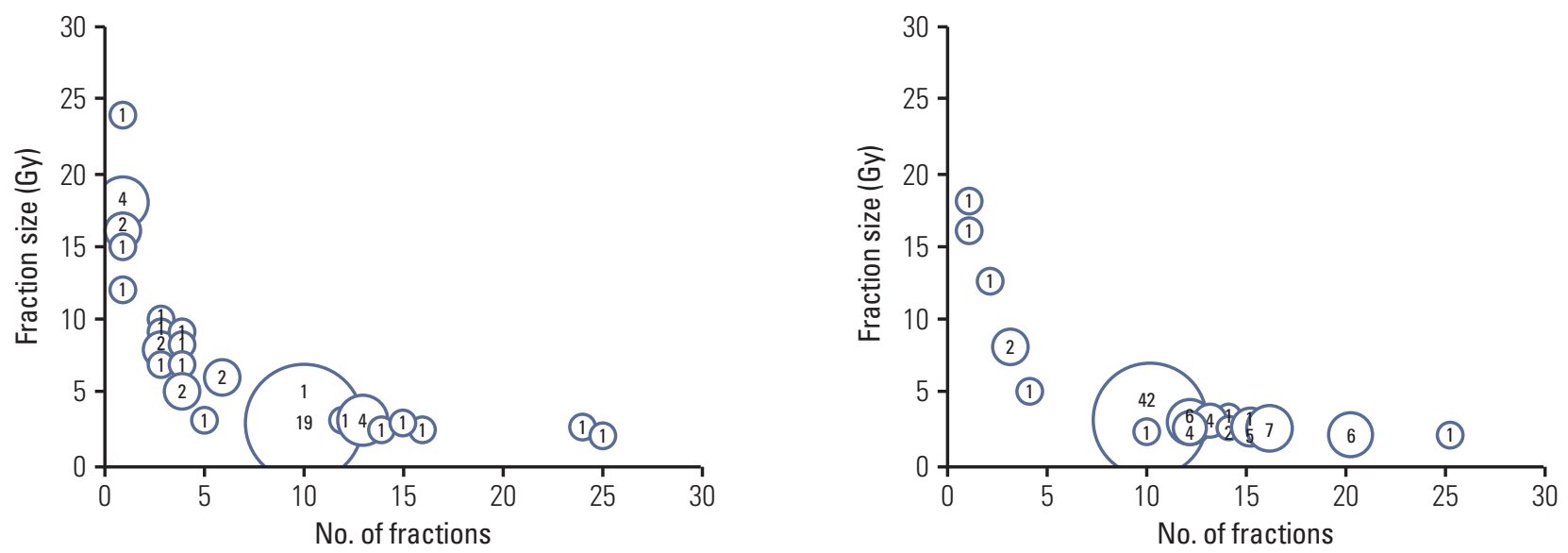

Fig. 2. Practice pattern changes from 2009 to 2014 in similar scenarios with favorable life expectancy. Preference for radiotherapy fractionation in the case with a Karnofsky performance status of 90 and solitary T9 level spine metastasis of renal cell carcinoma with stable disease of both lung and adrenal metastases after target therapy in the current study (A). A similar case of breast cancer with T6-7 spine only metastasis that had excellent performance status from the 2009 study (B) is displayed. Hypofractionation representing stereotactic ablative body radiotherapy was significantly increased compared with the results of the 2009 study, but the multiple fractionation scheme still accounted for a large proportion of answers.

ondents preferred ten or more fractions. This was consistent with the results of the previous study. As suggested in the previous study, the reimbursement system might be the main reason for the preference for multiple fractionation. Training experience might be an important reason for the observed practice pattern preferences, as indicated in a Cana- 
dian PCS [15].

In a favorable spine metastasis case, there was a tendency toward small fractionation preference, which is significantly changed from the 2009 study. This may be related to the usage of advanced technique RT. As results of SABR on spine metastasis have accumulated [22-24], the preference for SABR has increased significantly since 2009; in particular, it is more frequent in institutions where more than five radiation oncology specialists work. In general, working with a greater number of radiation oncologist specialists indicates that he or she works in a large training institution in Korea.

Therefore, SABR on spine metastasis with favorable prognosis will be rapidly chosen more often by radiation oncologists because the practice pattern is clearly affected by training experience as results of Canadian PCS.

Despite the use of multiple fractions at high doses or use of SABR in spine metastasis, a large proportion of patients will not benefit in terms of quality of life or survival period. For stratification of risk groups to optimize a palliative approach to spine metastasis, a reliable life expectancy table according to prognostic factors based on a large-scale multicenter study is needed [19-21]. To encourage more evidencebased practice on spine metastasis in Korea, guidelines from the KOSRO based on a reliable life expectancy table might be helpful.

An important limitation of the current study is that we could not guarantee the representativeness of the results because the study was performed by only one radiation oncologist from each institution participated, and one third did not respond to the questionnaires.

\section{Conclusion}

In conclusion, the current PCS demonstrated that multiple fraction RT is still preferred by a large proportion of Korean radiation oncologists, although there are many studies supporting the equivalence of a single fraction to multiple fractions in addition to the previous publication of the PCS study. To encourage more evidence-based practice in RT for spine metastasis, new guidelines for RT of spine metastasis might be needed.

\section{Electronic Supplementary Material}

Supplementary materials are available at Cancer Research and Treatment website (http://www.e-crt.org).

\section{Conflicts of Interest}

Conflict of interest relevant to this article was not reported.

\section{Acknowledgments}

This study was supported in part by a Samsung Medical Center grant (GF01130081) and the National Evidence-based Healthcare Collaborating Agency (NECA-A-09-002).

\section{References}

1. Chow E, Zeng L, Salvo N, Dennis K, Tsao M, Lutz S. Update on the systematic review of palliative radiotherapy trials for bone metastases. Clin Oncol (R Coll Radiol). 2012;24:112-24.

2. Crnalic S, Lofvenberg R, Bergh A, Widmark A, Hildingsson C. Predicting survival for surgery of metastatic spinal cord compression in prostate cancer: a new score. Spine (Phila Pa 1976). 2012;37:2168-76.

3. Douglas S, Schild SE, Rades D. Metastatic spinal cord compression in patients with cancer of unknown primary. Estimating the survival prognosis with a validated score. Strahlenther Onkol. 2012;188:1048-51.

4. Katagiri H, Takahashi M, Wakai K, Sugiura H, Kataoka T, Nakanishi K. Prognostic factors and a scoring system for patients with skeletal metastasis. J Bone Joint Surg Br. 2005; 87:698-703.

5. Rades D, Douglas S, Veninga T, Stalpers LJ, Bajrovic A, Rudat $\mathrm{V}$, et al. Prognostic factors in a series of 504 breast cancer patients with metastatic spinal cord compression. Strahlenther Onkol. 2012;188:340-5.

6. Tokuhashi Y, Uei H, Oshima M, Ajiro Y. Scoring system for prediction of metastatic spine tumor prognosis. World J Orthop. 2014;5:262-71.

7. Fairchild A, Barnes E, Ghosh S, Ben-Josef E, Roos D, Hartsell $\mathrm{W}$, et al. International patterns of practice in palliative radiotherapy for painful bone metastases: evidence-based practice? Int J Radiat Oncol Biol Phys. 2009;75:1501-10. 
8. Nakamura N, Shikama N, Wada H, Harada H, Nozaki M, Nagakura $\mathrm{H}$, et al. Patterns of practice in palliative radiotherapy for painful bone metastases: a survey in Japan. Int J Radiat Oncol Biol Phys. 2012;83:e117-20.

9. Popovic M, den Hartogh M, Zhang L, Poon M, Lam H, Bedard $\mathrm{G}$, et al. Review of international patterns of practice for the treatment of painful bone metastases with palliative radiotherapy from 1993 to 2013. Radiother Oncol. 2014;111:11-7.

10. Wu JS, Kerba M, Wong RK, McKimmon E, Eigl B, Hagen NA. Patterns of practice in palliative radiotherapy for painful bone metastases: impact of a regional rapid access clinic on access to care. Int J Radiat Oncol Biol Phys. 2010;78:533-8.

11. DeSantis C, Siegel R, Bandi P, Jemal A. Breast cancer statistics, 2011. CA Cancer J Clin. 2011;61:409-18.

12. Korean Breast Cancer Society. Breast Cancer White Paper 2014. Seoul: Korean Breast Cancer Society; 2014.

13. Bydon M, De la Garza-Ramos R, Bettagowda C, Gokaslan ZL, Sciubba DM. The use of stereotactic radiosurgery for the treatment of spinal axis tumors: a review. Clin Neurol Neurosurg. 2014;125:166-72.

14. Chow E, Harris K, Fan G, Tsao M, Sze WM. Palliative radiotherapy trials for bone metastases: a systematic review. J Clin Oncol. 2007;25:1423-36.

15. Olson RA, Tiwana MS, Barnes M, Kiraly A, Beecham K, Miller $\mathrm{S}$, et al. Use of single- versus multiple-fraction palliative radiation therapy for bone metastases: population-based analysis of 16,898 courses in a Canadian province. Int J Radiat Oncol Biol Phys. 2014;89:1092-9.

16. Chung Y, Koom WS, Ahn YC, Park HC, Kim HJ, Yoon SM, et al. A survey of patterns of practice on palliative radiation therapy for bone metastasis in Korea. J Cancer Res Clin Oncol.
2013;139:2089-96.

17. Dy SM, Asch SM, Naeim A, Sanati H, Walling A, Lorenz KA. Evidence-based standards for cancer pain management. J Clin Oncol. 2008;26:3879-85.

18. van den Hout WB, van der Linden YM, Steenland E, Wiggenraad RG, Kievit J, de Haes $H$, et al. Single- versus multiplefraction radiotherapy in patients with painful bone metastases: cost-utility analysis based on a randomized trial. J Natl Cancer Inst. 2003;95:222-9.

19. Leithner A, Radl R, Gruber G, Hochegger M, Leithner K, Welkerling $\mathrm{H}$, et al. Predictive value of seven preoperative prognostic scoring systems for spinal metastases. Eur Spine J. 2008;17:1488-95.

20. Wibmer C, Leithner A, Hofmann G, Clar H, Kapitan M, Berghold A, et al. Survival analysis of 254 patients after manifestation of spinal metastases: evaluation of seven preoperative scoring systems. Spine (Phila Pa 1976). 2011;36:1977-86.

21. Zadnik PL, Hwang L, Ju DG, Groves ML, Sui J, Yurter A, et al. Prolonged survival following aggressive treatment for metastatic breast cancer in the spine. Clin Exp Metastasis. 2014;31:47-55.

22. Chang EL, Shiu AS, Mendel E, Mathews LA, Mahajan A, Allen PK, et al. Phase I/II study of stereotactic body radiotherapy for spinal metastasis and its pattern of failure. J Neurosurg Spine. 2007;7:151-60.

23. Gerszten PC, Burton SA, Ozhasoglu C, Welch WC. Radiosurgery for spinal metastases: clinical experience in 500 cases from a single institution. Spine (Phila Pa 1976). 2007;32:193-9.

24. Lee S, Chun M, Lee M. Stereotactic body radiotherapy for solitary spine metastasis. Radiat Oncol J. 2013;31:260-6. 